\title{
The evolving dynamic response of a four storey reinforced concrete structure during construction
}

\author{
A. Devin* and P.J. Fanning \\ School of Architecture, Landscape \& Civil Engineering, University College Dublin, Belfield, Dublin, Ireland
}

\begin{abstract}
Structures include elements designated as load bearing and non-load bearing. While non-load bearing elements, such as facades and internal partitions, are acknowledged to add mass to the system, the structural stiffness and strength is generally attributed to load bearing elements only. This paper investigates the contribution of non-load bearing elements to the dynamic response of a new structure, the Charles Institute, in the grounds of University College Dublin (UCD) Ireland. The vertical vibration response of the first floor and the lateral response at each floor level were recorded at different construction stages. The evolution of the structural response as well as the generation of a finite element (FE) model is discussed. It was found that the addition of the non-load bearing facades increased the first floor natural frequency from $10.7 \mathrm{~Hz}$ to $11.4 \mathrm{~Hz}$, a change of approximately $+6.5 \%$. Similarly these external facades resulted in the first sway mode having its frequency increased by $6 \%$. The subsequent addition of internal partitions, mechanical services and furnishings resulted in the floor natural frequency reducing to 9.2 Hz. It is concluded that external facades have the net effect of adding stiffness and the effect of internal partitions and furnishings is to add mass. In the context of finite element modelling of structures there is a significant challenge to represent these non-structural elements correctly so as to enable the generation of truly predictive FE models.
\end{abstract}

Keywords: Operational modal analysis, frequency domain decomposition, non-structural elements

\section{Introduction}

A building consists of a primary structural system which is designed to resist a variety of loads. In addition to this primary system there are so-called "non-structural" elements or secondary systems. These elements include such components as cladding panels, mechanical services, internal partitions and furniture. Non-structural elements are usually attached to the primary structural system using connections designed to prevent load transfer from the primary structural system. It is therefore usually assumed that non-structural elements are detached from the primary structural system and do not affect the stiffness and dynamic response of structures.

However recent studies of the actual dynamic response of buildings have highlighted that non-structural components can in fact have a significant impact on the stiffness and dynamic response of structures. Gaiotti [1] found that this was the case for pre-cast cladding panels. It was found that their connection design caused panels to attract unexpected forces when the primary structural system was loaded laterally. The pre-cast panels unintentionally braced the structure, increasing the lateral stiffness of the structure tested. Falati [2], in her study of pre-cast flooring concluded that false flooring caused a reduction of the natural frequency of pre-cast concrete slabs due to the addition of mass. The inclusion of full-height internal partitions, however, had a significant impact on both the natural frequencies and damping of the structure. Miskovic et al. [3] analysed the effect of full-height non-structural partitions on the modal properties of two nominally identical floors in a steel-concrete composite structure. It was found

*Corresponding author: A. Devin, University College Dublin, School of Architecture, Landscape \& Civil Engineering, Belfield, Dublin 4, Ireland. E-mail: austin.devin@ucd.ie. 
that there was a difference in both natural frequencies and mode shapes of the floors which could only be attributed to the differing layouts of non-structural partitions. The differing layouts caused a $6 \%$ difference on average in natural frequencies.

$\mathrm{Li}$ et al. [4] analysed numerically the effect of non-structural elements on the lateral resistance of structures. It was found that there was a discrepancy between the theoretical and finite element analyses; this was deduced to be due to the added stiffness caused by the contact between the infill walls and structural frame. It was also found that infill walls can account for $60 \%$ of additional stiffness in the lateral direction. Su et al. [5] investigated the influence of non-structural components on the lateral stiffness of three tall buildings using full-scale ambient vibration measurements. It was concluded, with the aid of Finite Element (FE) models, that non-structural elements were the largest contributors to structural stiffness compared to other parameters added to the initial bare frame FE model. These other parameters included a modified Young's Modulus for reinforced concrete, the inclusion of secondary beams and the addition of flexible floor diaphragms.

The analysis of these structures highlighted the significant impact non-structural elements can have on the dynamic response of structures. Such findings indicate that an accurate representation of buildings cannot be made without explicit consideration of non-structural elements. Hence the development of methods to accurately represent non-load bearing elements in complete FE models of structures, validated with experimental data from in-service buildings, is important in order to assist designers in accurately predicting the dynamic properties of finished structures.

In this paper the effect of non-structural elements as they are added to a bare frame structure is presented. To track the contribution of non-structural elements, as the structure evolved toward completion, vibration response data was recorded at three stages so that an accurate picture of the effect of different non-structural components could be assessed.

\section{Test structure}

The Charles Institute on the University College Dublin (UCD) campus in Ireland is a four storey reinforced concrete frame office building (Fig. 1). Structurally it consists of two-way spanning flat slabs, $0.3 \mathrm{~m}$ thick, supported on $0.4 \mathrm{~m}$ square columns positioned on a $7.5 \mathrm{~m} \times 6.6 \mathrm{~m}$ grid. The lateral load resisting system is made up of a number of reinforced concrete stairwells, lift cores and service ducts with wall thicknesses of $0.2 \mathrm{~m}$. Each floor level is divided into laboratories and office accommodation using light-weight non-structural partitions consisting of plasterboard with a thickness of $12.5 \mathrm{~mm}$ and an approximate mass of $47 \mathrm{~kg} / \mathrm{m}$, supported on lightweight metal studs. Exterior wall cladding consisted of large polished Chinese black basalt panels, $40 \mathrm{~mm}$ thick, supported on galvanised steel rectangular sections fixed to the concrete slab above and below at $400 \mathrm{~mm}$ centres. The polished Basalt had an estimated mass of $430 \mathrm{~kg} / \mathrm{m}$.

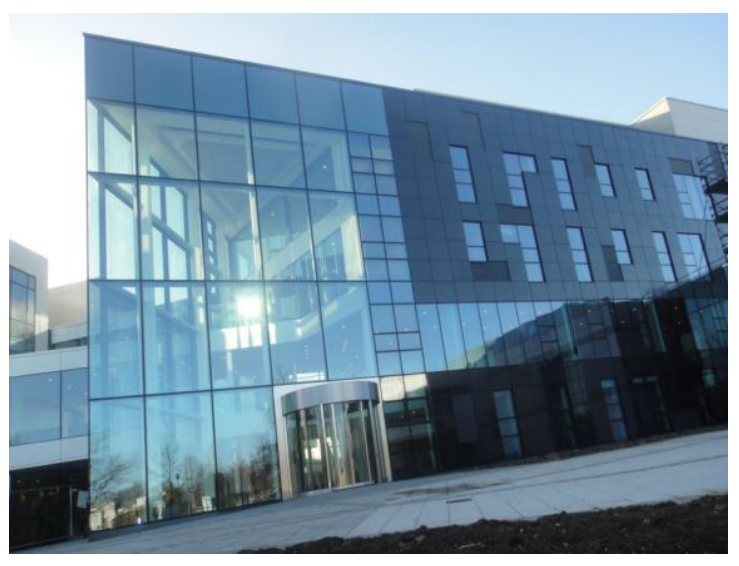

Fig. 1. Completed Charles Institute. 
The selection of sensor locations for recording of the vertical response of the first floor slab, numbered 1 to 18 in Fig. 2, was aided by a preliminary FE model so that a broad range of relevant mode shapes could be identified. The lateral response was recorded at sensor location 14 for test visit 1 (TV1) and sensor 18 for TV2 for each floor level. The different testing locations were required due to construction constraints during TV2. Test visits, TV1 to TV3, were made at three different stages of construction:

TV1: At the completion of the reinforced concrete frame.

TV2: Once cladding elements were added to two sides of the frame along gridlines A and B as shown in Fig. 2.

TV3: At building completion.

The internal partition layout as added between TV2 and TV3 is displayed in Fig. 3. Dotted lines highlight partitions added to the floor below.

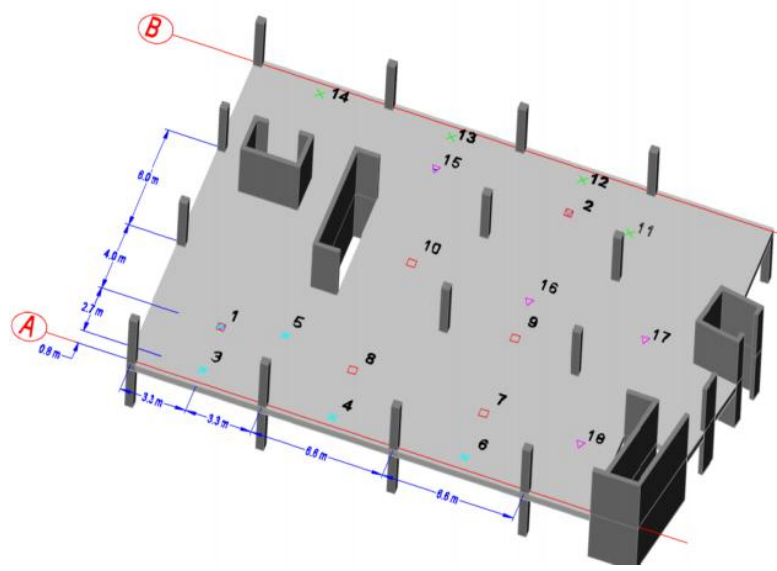

Fig. 2. Sensor locations: $1-18$.

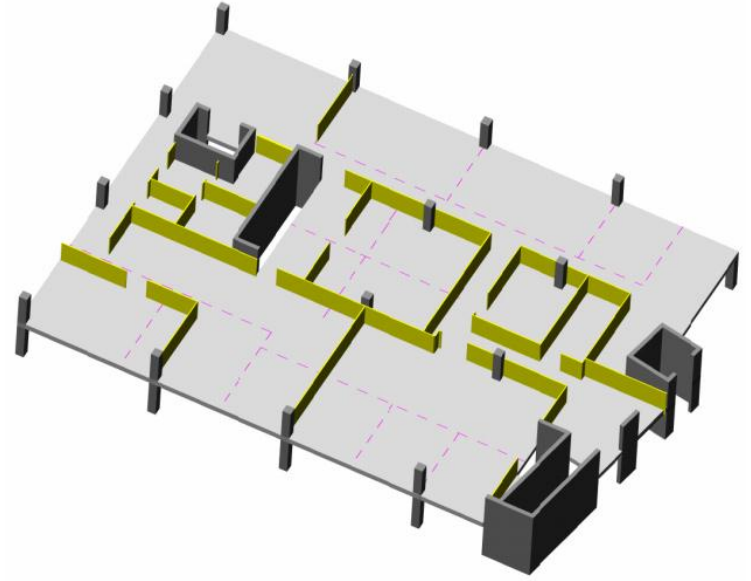

Fig. 3. First floor internal partition layout.

\section{Ambient response analysis}

Piezotronics accelerometers were used to measure the vibration response of the structure. No specific excitation was provided and the response measured was due to prevailing environmental conditions and limited construction activity associated with personnel carrying out secondary structural fixings. The sensors had a sensitivity of $700 \mathrm{mV} / \mathrm{g}$ and a measurement range of $\pm 3 \mathrm{~g}$. ENDEVCO amplifiers were used to scale the signals which were then recorded on a TEAC LX-10 Recording Unit. The ambient acceleration response of the structure was recorded for 10 minutes at a sampling rate of $12 \mathrm{kHz}$. Datasets of such length are considered more than adequate for structures with natural frequencies in the range of 5 to $30 \mathrm{~Hz}$ [6]. The recorded data sets were detrended using a linear baseline correction and cosine tapered to avoid data leakage. Data was then filtered using a low pass filter with an upper bound limit of $40 \mathrm{~Hz}$. The data sets were then post-processed and converted to the Frequency Domain using Frequency Domain Decomposition (FDD). Matlab algorithms were generated for data set preparation and the FDD.

Frequency Domain Decomposition utilises the fact that modes can be reasonably estimated from the spectral densities of analogue signals as long as the assumptions of white noise input and a lightly damped test structure are satisfied [7]. Peeters and Ventura [8] highlighted that in practical applications this theoretical assumption of white noise is not strict; as long as the input spectrum is quite flat and does not contain harmonics it will lead to satisfactory output-only modal analysis results.

The first step of the FDD process is to combine the multiple channel vibration data into a Power Spectral Density (PSD) matrix using a number of discrete Fourier Transforms [9]. This PSD matrix is then decomposed using Singular Value Decomposition (SVD) which represents the PSD matrix as the product of three separate matrices, $U$, the unitary matrix, $S$, the matrix of singular values and $V$, a transpose matrix. The values within the singular value matrix represent $n$ single degree of freedom models which describe the system in the frequency domain, where $n$ is 
the number of channels of vibration data. The respective modal amplitudes at each singular value are extracted from the $U$ matrix and form an approximation of the system's mode shapes.

\section{FE modelling and modal parameters identified}

\subsection{FE modelling}

A detailed 3D FE model of the building, shown in Fig. 4, was created in ANSYS according to final construction drawings using SHELL63 elements to model the slabs and shear cores and BEAM4 elements to model the columns.

The concrete mix specified for this building was C30/37 per Eurocode 2 [10]. Mehta and Monteiro [11] state that the dynamic modulus of elasticity for medium-strength concrete is $30 \%$ higher than its static modulus. Hence a dynamic modulus of elasticity of $48.5 \mathrm{GPa}$ was assigned to the concrete. In respect of the Poisson's ratio: Bamforth et al. [12] recommend 0.2; Neville [13] concluded that the Poisson's ratio value for concrete is dependent on the properties of the aggregate used but is typically in the range 0.17 to 0.20 ; while Mehta and Monteiro [11] confirm this in recommending a value between 0.15 and 0.2 . The structure's natural frequencies were found not to be overly sensitive to the Poisson's ratio and the results reported are for a value of 0.18 . The concrete density specified was $24.5 \mathrm{~N} / \mathrm{mm}^{2}$.

Seventy natural frequencies were predicted by FE modal analysis in the range of $3 \mathrm{~Hz}$ to $30 \mathrm{~Hz}$ these included global sway and torsion modes as well as local vertical bending modes of each of the floors. The first lateral sway mode shape is shown in Fig. 5.

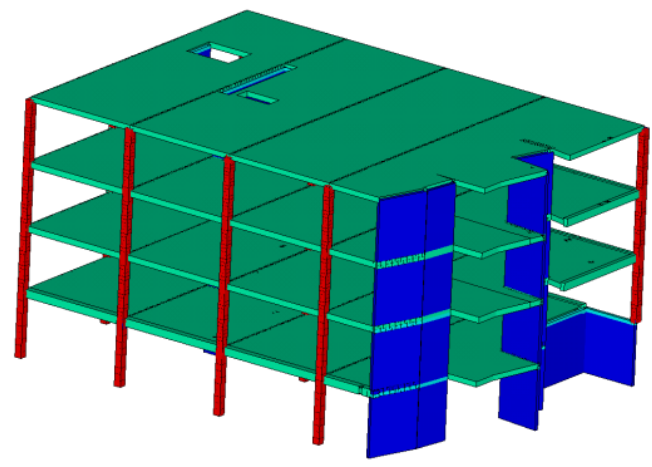

Fig. 4. 3D FE model of the Charles Institute frame.

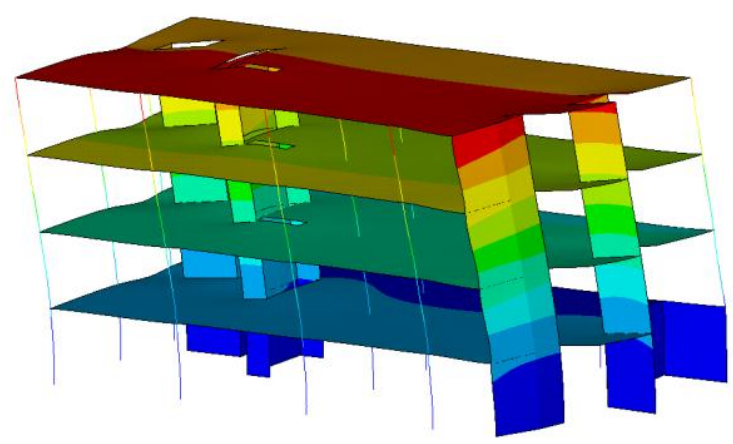

Fig. 5. Global sway mode of structure at $2.9 \mathrm{~Hz}$.

\subsection{Analysis of TV1 vertical response data}

The singular value (SV) plot of the vertical acceleration response for the first floor, recorded during TV1, is plotted in Fig. 6; the first natural frequency occurs at $10.7 \mathrm{~Hz}$. In addition there are clusters of natural frequencies in the ranges from $13-15 \mathrm{~Hz}$ and $21-24 \mathrm{~Hz}$ and also frequencies between and above these ranges. This wide distribution of frequencies is expected due to the regular pattern of the slab system between supporting columns and shear walls. The primary modes extracted for the first floor slab are primarily vertical bending modes characterised by vibration of the slab in varying bays between columns.

Visual inspection and comparison with the mode shapes extracted from the vertical response data sets during TV1, the bare frame stage, and the FE model using the Modal Assurance Criterion (MAC) values highlighted the most suitable mode shapes to compare to the experimental results. Table 1 summarises this comparison of the natural frequencies of the first floor and MAC values extracted from TV1 to those of the 3D FE model. Only modes with MAC values of 0.7 and above are retained. The first three mode shapes extracted from the ambient test data, occurring at natural frequencies of $10.7,12.4$ and $14.1 \mathrm{~Hz}$, had a good correlation to those of the 3D FE model, the 
lowest MAC value being 0.84 and the highest percentage difference being 5.7\%. This indicates a very good correlation between experimental and numerical results for these first three modes, as can be seen visually for the first mode in Fig. 7. Test and numerical results yield a mode shape characterised by bending of the slab in the regions of sensors 1-8 (see Fig. 2).

Table 1

Comparison of natural frequencies from Floor Level 1 for TV1 and the 3D FE model

\begin{tabular}{ccccc}
\hline & TV1 $(\mathrm{Hz})$ & FE model $(\mathrm{Hz})$ & MAC & Diff $(\%)$ \\
\hline & 10.7 & 11.1 & 0.84 & 3.7 \\
2 & 12.4 & 12.8 & 0.91 & 3.2 \\
3 & 14.1 & 13.3 & 0.91 & -5.7 \\
4 & 27.3 & 27.2 & 0.74 & -0.3 \\
5 & 29.8 & 27.3 & 0.75 & -8.2 \\
6 & 35.1 & 28.2 & 0.85 & -19.7 \\
7 & 35.3 & 29.2 & 0.88 & -17.3 \\
\hline
\end{tabular}

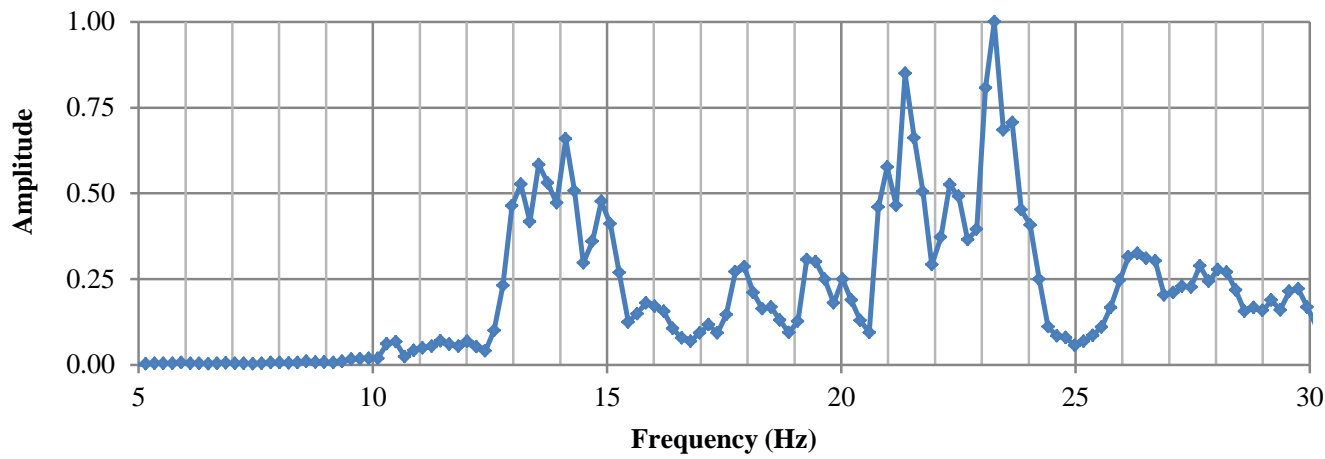

Fig. 6. First singular value plot in the Frequency Domain for TV1.

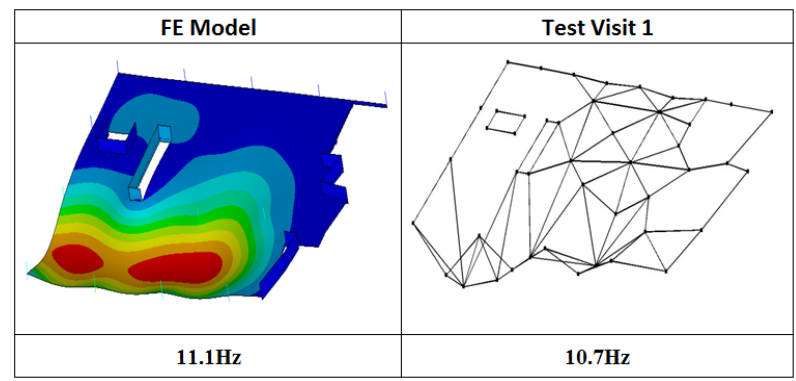

Fig. 7. Comparison of first mode of vibration of the slab for the 3D FE model and TV1.

Other clearly identified modes include those at $35.1 \mathrm{~Hz}$ and $35.3 \mathrm{~Hz}$, with MAC values of 0.85 and 0.88 respectively, although the error in frequencies is larger for these higher modes. While for mode 4 there is a good agreement of frequencies, the MAC comparison indicates non-identical modes. The reason for this is not obvious from the data although it is possible that the low MAC value is due to spatial aliasing due to low sensor density rather than a poor mode shape correlation.

Given these comparisons the three most sensible modes to concentrate on for further enhancement of the FE model, in subsequent work, which will include subsystems of elements to represent the non-load bearing elements, are the lower modes at $10.7 \mathrm{~Hz}, 12.4 \mathrm{~Hz}$ and $14.1 \mathrm{~Hz}$ respectively. 


\subsection{Comparison of modal parameters from TV1 to TV2 to TV3}

The same test procedure for the vertical response of the first floor was reproduced in visits TV2 and TV3. It was found that ambient excitation levels diminished as non-structural elements were added, decreasing from $1.1 \mathrm{~cm} / \mathrm{s}^{2}$ in TV1 to $0.66 \mathrm{~cm} / \mathrm{s}^{2}$ in TV2 and finally to $0.46 \mathrm{~cm} / \mathrm{s}^{2}$ in TV3. This indicated a less responsive floor as the structure evolved to completion. SV plots for the vertical response of the first floor during the three test visits are compared in Fig. 8. There is a clear change in response between test visits. For TV2 the natural frequency close to $10.7 \mathrm{~Hz}$ is retained but the location of the clusters is shifted upwards - indicating stiffening of the response in these modes this is consistent with the reducing magnitude of vibration levels observed. From TV2 to TV3 there is a more complex change in the behaviour of the structure, the distinct clusters are no longer evident and the lowest frequency recorded reduces to just over $9 \mathrm{~Hz}$. Significantly there is generally a reduction in frequencies from TV2 to TV3.

MAC values arising from a comparison of mode shapes extracted from TV1 and TV2 are shown in Table 2. For the first three modes the respective MAC values are $0.81,0.77$ and 0.9 respectively. This would indicate that the modes are substantially the same for the two test visits and that a stiffening response between TV1 and TV2 is being observed as the structure evolves towards completion. The increases in frequency for these three modes, relative to the TV1 values, are $6.5 \%, 8.9 \%$ and $8.5 \%$ respectively. It is noted also that there is strong coupling between the two mode sets for the first three TV1 modes and the first four TV2 modes. This is believed to be due to the sensor resolution being limited and each of these modes, differentiated more clearly in the FE model, being slightly different slab bending modes. There is also a high correlation between the modes which occurred at $21.4 \mathrm{~Hz}$ and $24.4 \mathrm{~Hz}$ for TV1 and TV2 respectively, equating to a frequency increase of $13 \%$.

Table 2

Matrix of MAC values arising from a comparison of first floor slab modes from TV1 to TV2

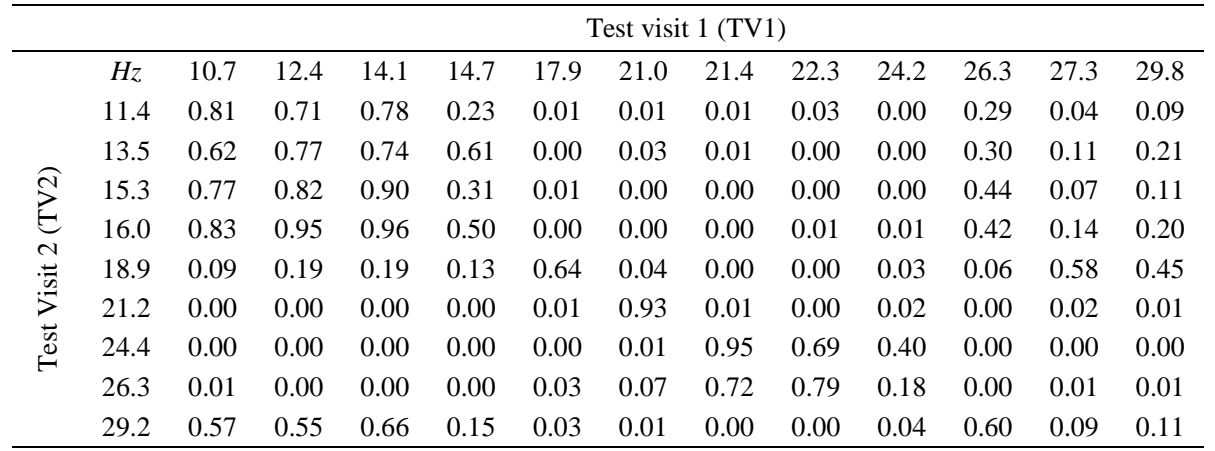

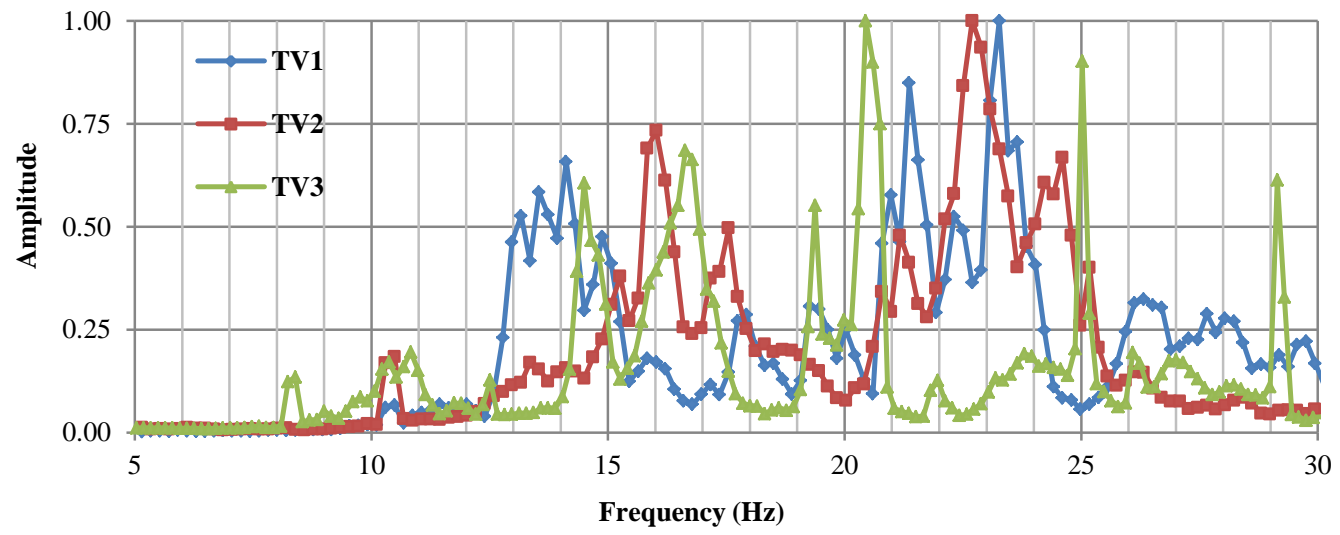

Fig. 8. First singular value plots of the vertical response of the first floor during TV1, TV2 and TV3. 
Table 3 shows the matrix of MAC values arising from a comparison of mode shapes extracted from TV2 and TV3. The natural frequencies for TV3 are in the range $9 \mathrm{~Hz}$ to $22 \mathrm{~Hz}$. The first four modes, as with the comparison of TV1 and TV2, have high correlation of MAC values. This indicates that once again while the natural frequencies have changed, the actual mode shapes themselves remain quite similar at low frequencies. At the time of TV2, cladding panels had been added to Gridlines A and B (Fig. 2) only, while the building had been completed and fully furnished, ready for occupation by the time of TV3.

Table 3

Matrix of MAC values arising from a comparison of first floor slab modes from TV2 to TV3

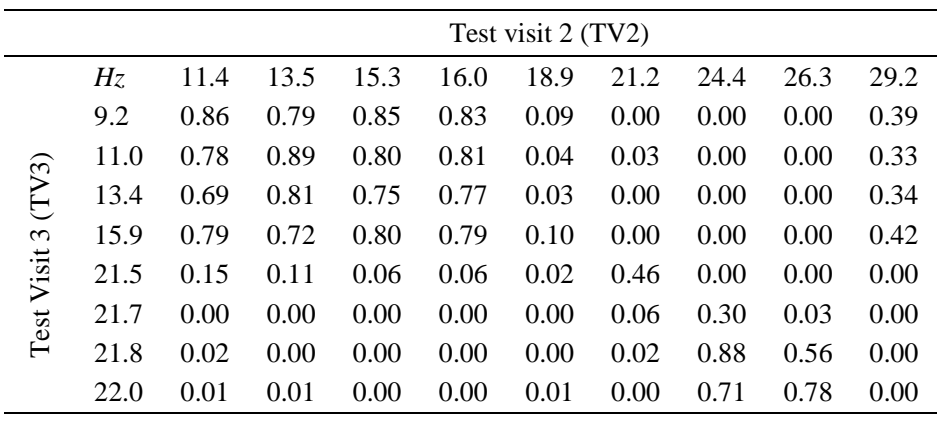

\subsection{Lateral response of structure}

The lateral ambient response of the building was recorded during TV1 and TV2. Due to limited accessibility to all floors during construction, accelerometers were only placed at sensor locations 14 and 18 on each floor and lateral response was measured in the direction perpendicular to gridline B (Fig. 2).

The lateral natural frequencies of the structure extracted during TV1 and TV2, along with the 3D FE model results, are summarised in Table 4. Given the low number of sensors, and to confirm that the frequencies identified are indeed associated with the lowest lateral modes, the first TV1 mode shape is compared with the first mode shape from the 3D FE model in Fig. 9. The modes compare well despite the FE model first frequency being lower than that measured on site. This discrepancy may be due to the fact there is a walkway between the Charles Institute and an adjacent building in this area which is not accounted for in the current 3D FE model. Comparing TV1 and TV2 there is an increase of $5 \%$ in the first natural frequency, which increases from $3.8 \mathrm{~Hz}$ in TV1 to $4.0 \mathrm{~Hz}$ in TV2. This shift in frequency is attributed to the addition of cladding panels, bracing the structure laterally, and perpendicular to the direction of response measured. No change in frequencies associated with the higher two lateral modes was measured. Due to construction constraints at the times of TV1 and TV2 the sensor locations for the lateral tests differed and therefore direct comparison of mode shapes extracted from these lateral tests is not possible.

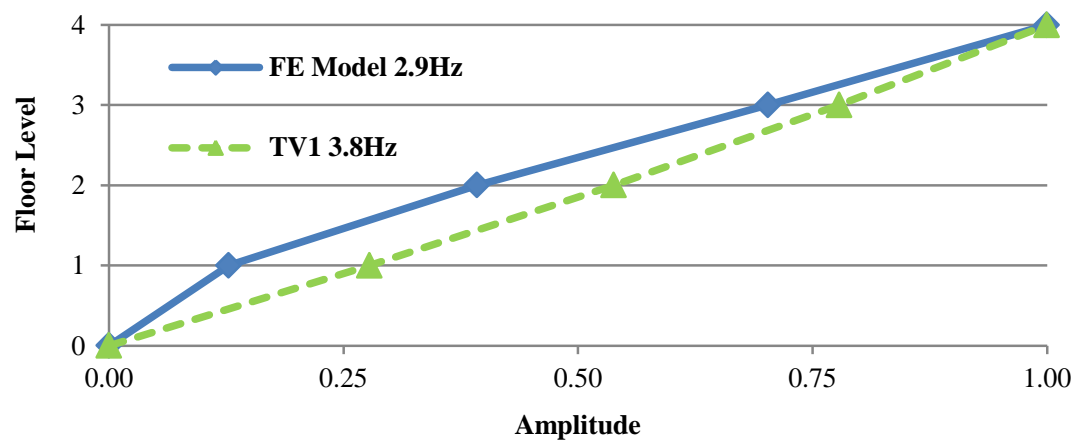

Fig. 9. Comparison of the first mode shapes from TV1 and the FE model. 
Table 4

Summary of natural frequencies extracted from lateral response data

\begin{tabular}{cccccc}
\hline FE model $(\mathrm{Hz})$ & TV1 $(\mathrm{Hz})$ & Diff $(\%)$ & TV1 $(\mathrm{Hz})$ & TV2 $(\mathrm{Hz})$ & Diff $(\%)$ \\
\hline 2.9 & 3.8 & $24 \%$ & 3.8 & 4.0 & $5 \%$ \\
10.8 & 10.5 & $-3 \%$ & 10.5 & 10.5 & $0 \%$ \\
20.3 & 20.8 & $3 \%$ & 20.8 & 20.8 & $0 \%$ \\
\hline
\end{tabular}

\section{Discussion of results}

The test sequence has shown an evolution in dynamic response from one visit to the next. The first visit was undertaken after completion of the load bearing frame. The evolution of response is thus directly attributable to the addition of non-load bearing elements and the final fit-out.

The 3D bare frame FE model of the Charles Institute Building correlated well with experimental data extracted from the bare frame of the structure (TV1). Whilst this model was useful in aiding the design of the sensor layouts for ab-initio testing it is not suitable for predicting the final response of the structure. The test findings indicate that truly representative FE models, of constructed structures, require inclusion of elements representing the contribution of non-structural components.

The change in the first three natural frequencies tracked between TV1 and TV2 is between $+6.5 \%$ and $+8.9 \%$. Between these two visits the structure had external facades added. The modes identified are largely comparable, as evidenced by the MAC values in Table 3. Thus the effect of the addition of external cladding, while obviously adding mass, was predominantly to stiffen the structure.

Between TV2 and TV3 the construction work was completed. All internal partitions and mechanical services had been fitted and the building was fully furnished and ready for occupancy. Between these two test visits frequencies were seen to reduce, with the slab's first mode of vibration reducing in frequency from $11.4 \mathrm{~Hz}$ to $9.2 \mathrm{~Hz}$. Frequencies associated with higher modes were also reduced. Clearly there is the potential that partitions may stiffen the slab locally but any stiffening effect, in this structure, is offset by the mass added between TV 2 and TV 3 and the net effect was a reduction in frequencies between these visits.

Taken collectively the measured data, at the three different construction stages, indicates that the net effect of external facades, certainly of the type used in this structure, where they are mounted on frames fitted to the floor slabs, is to stiffen the structure. On the other hand the net contribution of internal partitions, mechanical services and furnishings, which resulted in a reduction in frequencies, is more akin to the addition of mass.

\section{Conclusions}

Output-only ambient vibrations were recorded at the newly constructed Charles Institute on the University College Dublin campus at three stages of construction varying from a completed bare structural frame to a fully fitted out building ready for occupancy.

In general terms it is concluded that facades and partitions have an effect on the modal characteristics of structures and it is thus recommended that where vibration response serviceability compliance tests are required, that these be only executed on a structurally complete and fully furnished system.

Specifically it was found that non-structural elements, when added to this structure, had a significant impact on both the natural frequencies and vertical mode shapes of the floor slabs. The effect on the lateral modes of the structure was less pronounced.

Finally the tests showed that the contributions of different non-structural elements vary. External facades were found to result in a stiffening effect while internal partitions, mechanical services and furnishings were found to produce an effect more akin to that of additional mass on the structure. 


\section{Acknowledgments}

The authors wish to express their gratitude to the Irish Research Council for Science, Engineering and Technology for their financial support and WALLS Construction for access to the Charles Institute for the purpose of response measurements.

\section{References}

[1] R. Gaiotti and B. Stafford Smith, Stiffening of moment-resisting frame by precast concrete cladding, PCI Journal (1992), $72-84$

[2] S. Falati, The contribution of non-structural components to the overall dynamic behaviour of concrete floor slabs, PhD Thesis, New College, Oxford, 1999.

[3] Z. Miskovic, A. Pavic and P. Reynolds, Effect of full-height non-structural partitions on the modal properties of two nominally identical floors, Canadian Journal of Civil Engineering 36(7) (2009), 1121-1132.

[4] B. Li, G. Hutchinson and C. Duffield, The influence of non-structural components on tall building stiffness, The Structural Design of Tall and Special Buildings 10 (2009).

[5] R.K.L. Su, A.M. Chandler, M.N. Sheikh and N.T.K. Lam, Influence of non-structural components on lateral stiffness of tall buildings, Structural Design of Tall and Special Buildings 14(2) (2005), 143-164.

[6] M.Q. Feng, D.K. Kim, J.-H. Yi and Y. Chen, Baseline models for bridge performance monitoring, J Eng Mech 130(5) (2004), 562-569.

[7] R. Brincker, L. Zhang and P. Andersen, Modal identification of output-only systems using frequency domain decomposition, Smart Materials and Structures 10 (2001), 441-445.

[8] S. Peeters and C.E. Ventura, Comparative study of modal analysis techniques for bridge dynamic characteristics, Mechanical Systems and Signal Processing 17(5) (2003), 965-988.

[9] D.J. Ewins, Modal Testing: Theory and Practice, (2nd ed.), Research Studies Press, 2005.

[10] British Standards Institution. Eurocode 2: Design of concrete structures: British standard, London: BSi, 2008.

[11] P.K. Mehta and P.J.M. Monteiro, Concrete: Microstructure, Properties, and Materials, New York: McGraw-Hill, 2006.

[12] P. Bamforth, D. Chisholm, J. Gibbs and T. Harrison, Properties of Concrete for use in Eurocode 2, The Concrete Centre, UK, 2008.

[13] A.M. Neville, Properties of Concrete, (4th ed.), Essex: Longman Scientific and Technical, 1973. 

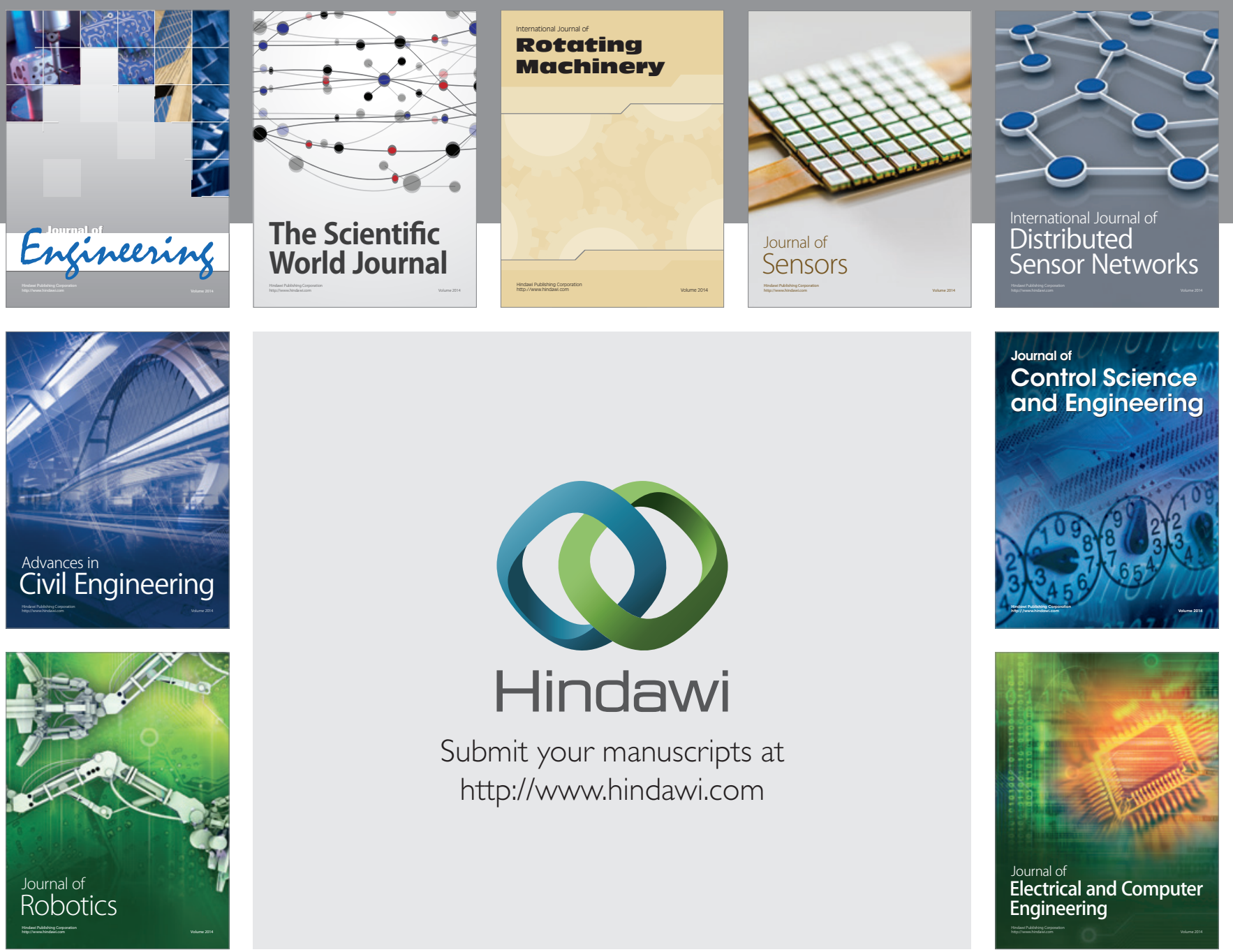

Submit your manuscripts at

http://www.hindawi.com
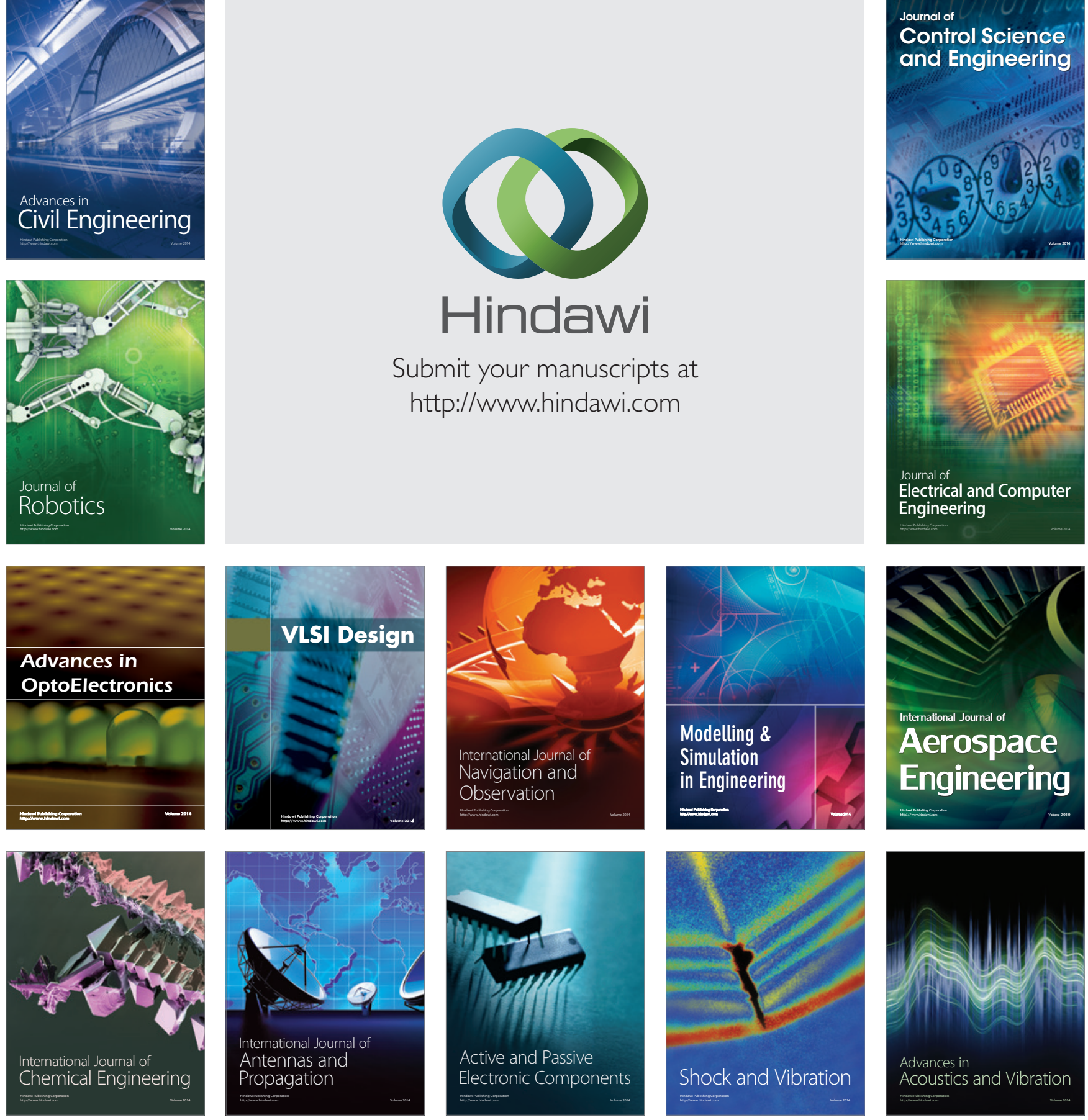